\title{
Combinatorial androgen receptor targeted therapy for prostate cancer
}

\author{
P Singh, A Uzgare, I Litvinov, S R Denmeade and J T Isaacs
}

Chemical Therapeutics Program, Sidney Kimmel Comprehensive Cancer Center, Johns Hopkins University, 1650 Orleans St. - CRB 162B, Baltimore, Maryland 21231, USA

(Requests for offprints should be addressed to J T Isaacs; Email: isaacjo @ jhmi.edu)

\begin{abstract}
Prostatic carcinogenesis is associated with changes in the androgen receptor (AR) axis converting it from a paracrine dependence upon stromal signaling to an autocrine-initiated signaling for proliferation and survival of prostatic cancer cells. This malignant conversion is due to gain of function changes in which the AR activates novel genomic (i.e. transcriptional) and non-genomic signaling pathways, which are not present in normal prostate epithelial cells. During further progression, additional molecular changes occur which allow these unique malignancy-dependent AR signaling pathways to be activated even in the low androgen ligand environment present following androgen ablation therapy. These signaling pathways are the result of partnering the AR with a series of other genomic (e.g. transcriptional co-activators) or non-genomic (e.g. steroid receptor co-activator (Src) kinase) signaling molecules. Thus, a combinatorial androgen receptor targeted therapy (termed CART therapy) inhibiting several points in the AR signaling cascade is needed to prevent the approximately 30,000 US males per year dying subsequent to failure of standard androgen ablation therapy. To develop such CART therapy, a series of agents targeted at specific points in the AR cascade should be used in combination with standard androgen ablative therapy to define the fewest number of agents needed to produce the maximal therapeutic antiprostate cancer effect. As an initial approach for developing such CART therapy, a variety of new agents could be combined with luteinizing hormone-releasing hormone analogs. These include: (1) $5 \alpha$-reductase inhibitors to inhibit the conversion of testosterone to the more potent androgen, dihydrotestosterone; (2) geldanamycin analogs to downregulate AR protein in prostate cancer cells, (3) 'bulky' steroid analogs, which can bind to AR and prevent its partnering with other co-activators/signaling molecules, and (4) small molecule kinase inhibitors to inhibit MEK, which is activated as part of the malignant AR signaling cascade.
\end{abstract}

Endocrine-Related Cancer (2006) 13 653-666

\section{Introduction}

Androgens are the major growth factors for the normal prostate, and its cognate receptor is fundamental for androgen signaling within the gland. Prostate cancers retain androgen receptor (AR) signaling pathways and thus are nearly universally responsive initially to androgen ablation therapy. Unfortunately, however, essentially all ablated patients eventually relapse. Owing to this relapse, androgen ablation therapy is not curative, no matter how complete the ablation is. There has been a major shift in the thinking concerning the role of $\mathrm{AR}$ in prostate cancer progression to this lethal stage. Since aggressive forms of androgen ablation (i.e. luteinizing hormone releasing (LHR) analogs plus anti-androgens plus chemical inhibition of adrenal androgen production) do not substantially increase the survival of prostate cancer patients above that produced by luteinizing hormone-releasing hormone (LHRH) analogs alone, it had been assumed that this therapeutic failure meant that $A R$ is no longer engaged in the lethal phase of the disease. A series of correlative and experimental data, however, do not support such a conclusion. With regard to the correlative data: (1) AR continues to be expressed in a significant subset of cells in metastatic tissue obtained at autopsy from androgen ablation-failing patients (Shah et al. 2004); (2) such AR expression is detectable within cell nuclei (Mohler et al. 2004, Shah et al. 2004); and (3) enhanced levels of AR mRNA and protein are the most consistent molecular markers 
correlated with the acquisition of an androgen ablation-resistant phenotype (Chen et al. 2004). With regard to experimental data, even within cancers in which only a subset of malignant cells continue to express AR, it has been documented that androgeninduced autocrine growth factor secreted from these AR-positive (i.e. pace maker) cancer cells stimulates the growth of AR-negative cancer cells in a paracrine manner (Nonomura et al. 1988). Also, the majority of in vitro prostatic cancer cell lines (e.g. LNCaP, LAPC4, LAPC-9, MDA-PC-2B, V-Cap, DuCap, etc.) established from patients failing androgen ablation continue to express AR (van Bokhoven et al. 2003) and if this expression is lowered by a variety of means (e.g. intracellular injection of anti-AR antibodies, anti-sense, siRNA, a hammerhead ribozyme, etc.), the proliferation of the 'androgen ablation-resistant' cancer cell lines is inhibited and the cell dies (Chen et al. 1998, Eder et al. 2002, Solit et al. 2002, Zegarra-Moro et al. 2002, Chen et al. 2004, Liao et al. 2005, Yang et al. 2005). Combining these correlative and experimental data has re-focused attention on how such androgen ablation-resistant cells use the AR to stimulate their proliferation and survival.

A growing body of data has documented that this is due to gain of function in the AR signaling pathways during the progression of prostatic cancer (Litvinov et al. 2003). This gain of function changes results in prostate cancer cells that are resistant to androgen ablation because they proliferate and survive without requiring physiological levels of androgen ligand. These changes produce malignancy unique signaling pathways that, while androgen ablation resistant, are still dependent upon AR (Litvinov et al. 2003). This AR dependency provides a therapeutic Achilles' heel for control of this devastating disease (Litvinov et al. 2003). The rationale for this statement is based on the following facts. First, the AR gene is located on the $\mathrm{X}$-chromosome, and thus males have only a single copy of this gene. Secondly, germline truncation mutations early in the first exon of the AR gene result in complete androgen-insensitivity syndrome because no expression of AR protein occurs in these patients. Although such complete androgen-insensitivity syndrome mutations prevent masculinization, they are not life threatening. This means that in prostate cancer patients with germline wild-type AR, systemic therapy that either selectively prevents AR expression or neutralizes its signaling ability should not be lethal to normal host tissues, except the male accessory sex tissues. These accessory sex tissues undergo regression by standard androgen ablation without affecting host survival. Therefore, such systemic AR-targeted therapy would have a restricted AR-dependent therapeutic index because blocking AR signaling while eliminating the metastatic prostate cancer cells remaining after androgen ablation would not be life threatening. Thus, prostate cancers should provide a paradigm for successful rational drug development based on this unique therapeutic index.

For such rational drug development, identification of the novel malignancy-acquired AR signaling pathways is critical. An understanding of the AR signaling in the normal prostate is required for such identification. Human prostatic glands are composed of a simple stratified epithelium containing a basal and luminal layer separated via basement membrane from a welldeveloped stromal compartment. The homeostatic maintenance of this prostatic epithelium is regulated via a hierarchical stem cell organization (Isaacs 1987), shown in Fig. 1. In the prostate epithelium, stem cells are rare and are located within the basal layer (i.e. $\leq 1 \%$ of basal cells are stem cells (Richardson et al. 2004)). Prostate stem cells proliferate rarely to renew the fraction of their progeny, which instead of remaining as uncommitted stem cells, enter a terminal maturation process in which several sequential stages have been identified phenotypically and morphologically (Litvinov et al. 2003). The earliest stage is termed a transitamplifying (TA) cell, which has a high proliferative potential and is located in the basal layer. These TA cells express very low to undetectable levels of AR protein and do not express prostatic differentiation marker proteins (e.g. prostate-specific antigen (PSA), human glandular kallikrein-2 (hK2), and prostate-specific membrane antigen (PSMA)) (Litvinov et al. 2003). While this subset of AR-negative TA cells does not respond directly to androgen, these cells do require critical levels of androgen-stimulated paracrine growth factors (i.e. andromedins) for their proliferation but not survival (Uzgare et al. 2004). The presently identified andromedins include fibroblast growth factor 7 (FGF-7) (Yan et al. 1992), FGF-10 (Lu et al. 1999, Nakano et al. 1999), and insulin-like growth factor-I (IGF-I) (Ohlson et al. 2006). These andromedins are produced by the occupancy of the AR by its ligand within prostate stromal cells (Gao \& Isaacs 1998, Gao et al. 2001, Kurita et al. 2001). These TA cells express the dominant-negative $\mathrm{N}$-terminal truncated form of the p53 related, p63 gene (i.e. $\Delta$ Np63 $\alpha$ isotype) within their nucleus and high levels of 'basal-specific' cytokeratins (i.e. keratin 5 and 14), glutathione-S-transferase-Pi isoform $\left(\mathrm{GST}-\mathrm{P}_{\mathrm{i}}\right)$, standard form of CD-44 (CD-44 $)$, transglutaminase type II (TGT-2), and involucrin, but only low levels of luminal-specific keratins 8 and 18 (Litvinov et al. 2003). Besides proliferating, these 


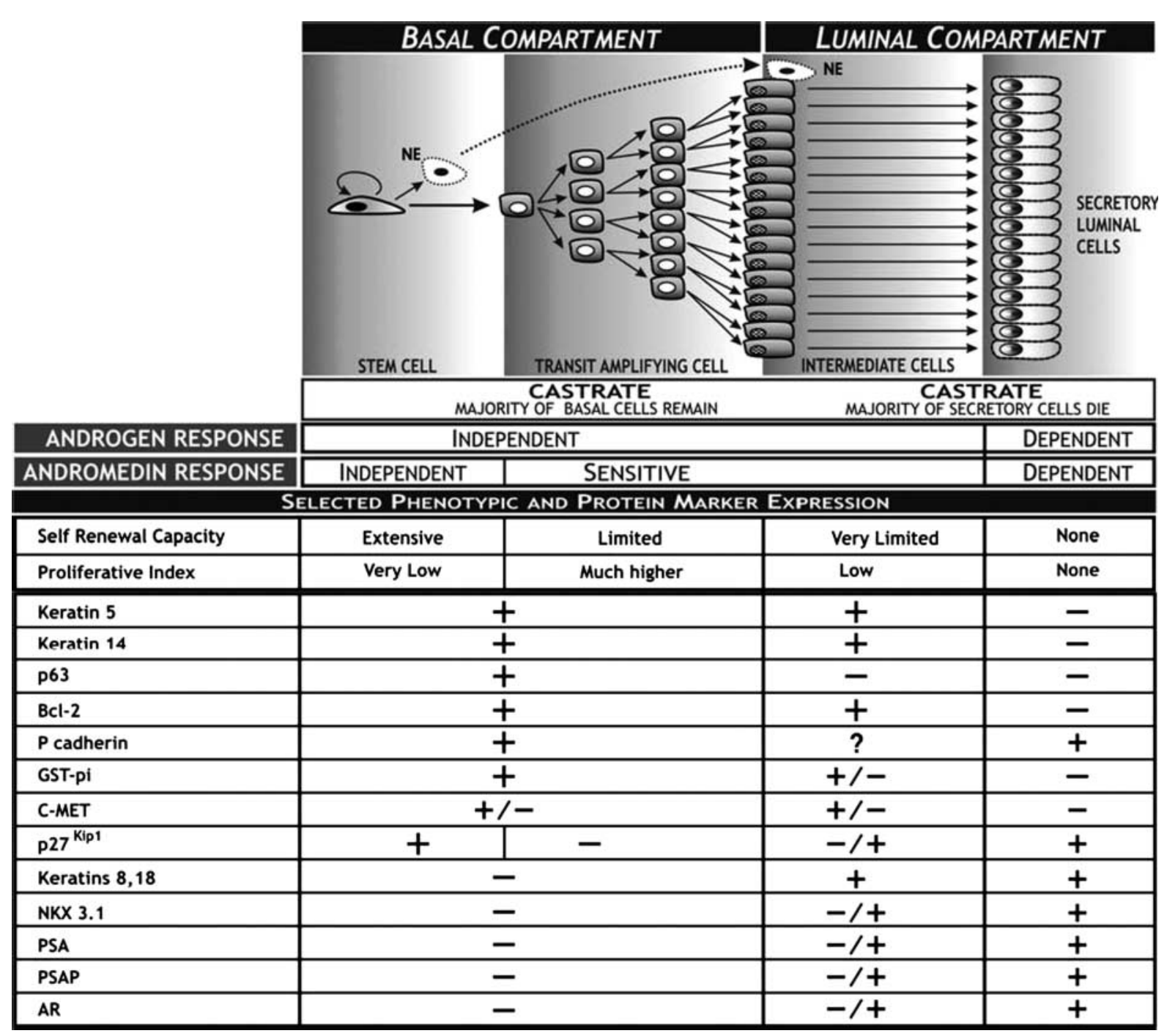

Figure 1 Stem cell model of prostatic epithelial cell compartmentalization. The prostate gland consists of a number of stem cell units that arise from one stem cell. Such a stem cell is located in the basal epithelial layer of the prostate and, upon division, gives rise to a population of transit-amplifying cells. The latter divide in the basal layer, and a fraction of them differentiate and move into the secretory luminal epithelial layer. As transit-amplifying cells differentiate and move into a secretory luminal layer from the basal layer, they acquire expression of a number of genetic markers, as indicated. Low-level retention of expression by a subset of transitamplifying (i.e. intermediate) cells; +, expression of marker; -, lack of detectable expression of marker; NE, Neuro-endocrine.

nuclear $\Delta$ Np63 $\alpha$ expressing TA cells undergo a process of maturation into 'basal intermediate' cells in the basal epithelial compartment (De Marzo et al. 1998, Tran et al. 2002, Garraway et al. 2003).

This maturation into basal intermediate cells involves the loss of expression of keratin 14 while maintaining the co-expression of basal-specific keratin 5 and luminal-specific keratins 8 and 18 , and $\Delta \mathrm{Np} 63 \alpha$, coupled with a decrease in their growth fraction (De Marzo et al. 1998, Tran et al. 2002, Garraway et al. 2003). The basal intermediate cells continued to mature with their gain of expression of prostate stem cell antigen (PSCA) protein and AR mRNA but not protein during their migration into the luminal layer to become 'luminal intermediate' cells (Tran et al. 2002). The luminal intermediate cells translate AR mRNA and thus express AR protein whose occupancy by androgen produces translocation of $\mathrm{AR}$ onto this nucleus where it binds to a specific DNA-response element of the promoters of specific differentiation genes (e.g. PSA, hK2 and PSMA) regulating their transcription. Due to this genomic effect, the 'intermediate' cells mature into fully differentiated, luminal secretory cells which express PSA, hK2 and PSMA but no longer express basal markers like keratins 5 and 14 and $\Delta \mathrm{Np} 63$. This terminal maturation is also associated 
with upregulation of the $\mathrm{p} 27^{\mathrm{Kip} 1}$ cyclin-dependent kinase inhibition protein and loss of proliferative ability (Waltregny et al. 2001). The mechanism for this upregulation in normal prostatic epithelial cells involves enhanced stability of the $\mathrm{p} 27^{\mathrm{Kip} 1}$ protein, secondary to AR-induced transcriptional repression of expression of the E3 ubiquitin ligase Skp2 involved in p2 $7^{\text {Kip1 }}$ degradation (Waltregny et al. 2001). While the engagement of nuclear AR in these luminal secretory cells regulates PSA, hK2 and PSMA transcription it does not regulate their survival. Instead, such survival requires adequate levels of the androgen-stimulated stromally derived andromedins (Gao \& Isaacs 1998, Gao et al. 2001, Kurita et al. 2001).

\section{Gain of function changes converts AR from a growth suppressor to an oncogene in prostate cancer cells}

Unlike the paracrine situation in the normal prostate in which such growth regulation is initiated by AR binding to genomic sequences in the nuclei of stromal cells, it is found during prostatic carcinogenesis that genomic AR binding within the transformed cells themselves activates this growth regulation. Because of these hard-wiring changes, there is a conversion from paracrine to autocrine AR signaling pathways in invasive prostate cancer (Gao \& Isaacs 1998, Gao et al. 2001). These gain of function hard-wiring changes pathologically allow androgen/AR complexes to bind to and enhance expression of survival and proliferation genes (i.e. 'malignant' andromedins, which are not necessarily the same andromedins (i.e. IGF-I, FGF-7, FGF-10) produced in the normal gland) that are physiologically not regulated by these AR complexes in either normal transit-amplifying or secretory luminal cells. Even with these hard-wiring changes, activation of these malignant-dependent growth-promoting (i.e. oncogenic) pathways can still require physiological levels of androgen for sufficient occupancy and binding of the dimerized AR within prostatic cancer cell nuclei to induce genomic (i.e. transcriptional) stimulation of their proliferation and survival. Such malignant cells depend upon physiological levels of circulating androgen as documented by the fact that lowering serum testosterone to $<0.5 \mathrm{ng} / \mathrm{ml}$ via LHRH analog-induced suppression of testicular testosterone production results in their death (Redding et al. 1992). Unfortunately, such physiologic androgen-dependent prostatic cancer cells undergo additional molecular changes in which AR interacts with partner proteins to generate genomic as well as non-genomic signaling even in the presence of low circulating serum testosterone levels produced by androgen ablation. Thus, these latter cells are not eliminated by standard androgen ablation (i.e. LHRH \pm anti-androgens) and their continuing growth eventually kills the patient. Presently, there is no curative therapy for these lethal androgen ablationresistant prostatic cancer cells.

To develop effective therapy, an understanding of how these cells develop resistance to androgen ablation is fundamental. There are several mechanisms, that have been identified for how such resistance to androgen ablation develops (Isaacs \& Isaacs 2004). These include the ability of the cancer cell to: (1) amplify AR signaling by metabolically converting the less potent testosterone via $5 \alpha$-reductase activity to the 10 times more potent $A R$ ligand, dihydrotestosterone (DHT) (Deslypere et al. 1992, Titus et al. 2005a, b); (2) enhance its level of AR protein so that even at the reduced level of androgen ligand remaining following androgen ablation, due to mass action, there are sufficient total molecules of ligandbound AR translocated to nuclei to initiate genomic (i.e. transcriptional) upregulation of 'malignant' andromedins even though the fraction of ligand-occupied AR still remains low (Chen et al. 2004); (3) enhanced levels of AR-transcriptional co-activators (e.g. p160 and p300), that 'forces' the ligand-unoccupied AR from an antagonist into its agonist conformation, thus activating ligand-independent genomic (i.e. transcriptional) effects to produce 'malignant' andromedins (Gregory et al. 2001, 2004, Debes et al. 2002, Culig et al. 2004). These malignant andromedins then bind to their appropriate plasma membrane cognate receptor generating survival and proliferation signaling; and 4) initiate AR binding to scaffolding protein complexes (e.g. modulator of nongenotropic activity of estrogen receptor (MNAR)steroid receptor co-activator ( $\mathrm{Src}$ ) kinase) activating the Src kinase ability to phosphorylate and activate the kinase of MEK resulting in a non-genomic kinase cascade signaling survival and proliferation (Castoria et al. 2004, Unni et al. 2004). The importance of this non-genomic Src/MEK kinase cascade is presently unresolved but treatment of human prostatic cancer cell lines in vitro with MEK inhibitor produces significant growth inhibitor but little cell death induction (Uzgare \& Isaacs 2004).

\section{Rationale for CART therapy}

Based upon this growing understanding of the multiple and often coordinated mechanisms for resistance to androgen ablation therapy, it is clear that a combinatorial approach to the simultaneous targeting of multiple AR pathways is required as a 'rational' approach to therapy. A summary of several of the possible sites for such a combinatorial AR targeted therapy, termed CART therapy, is presented in Fig. 2. 


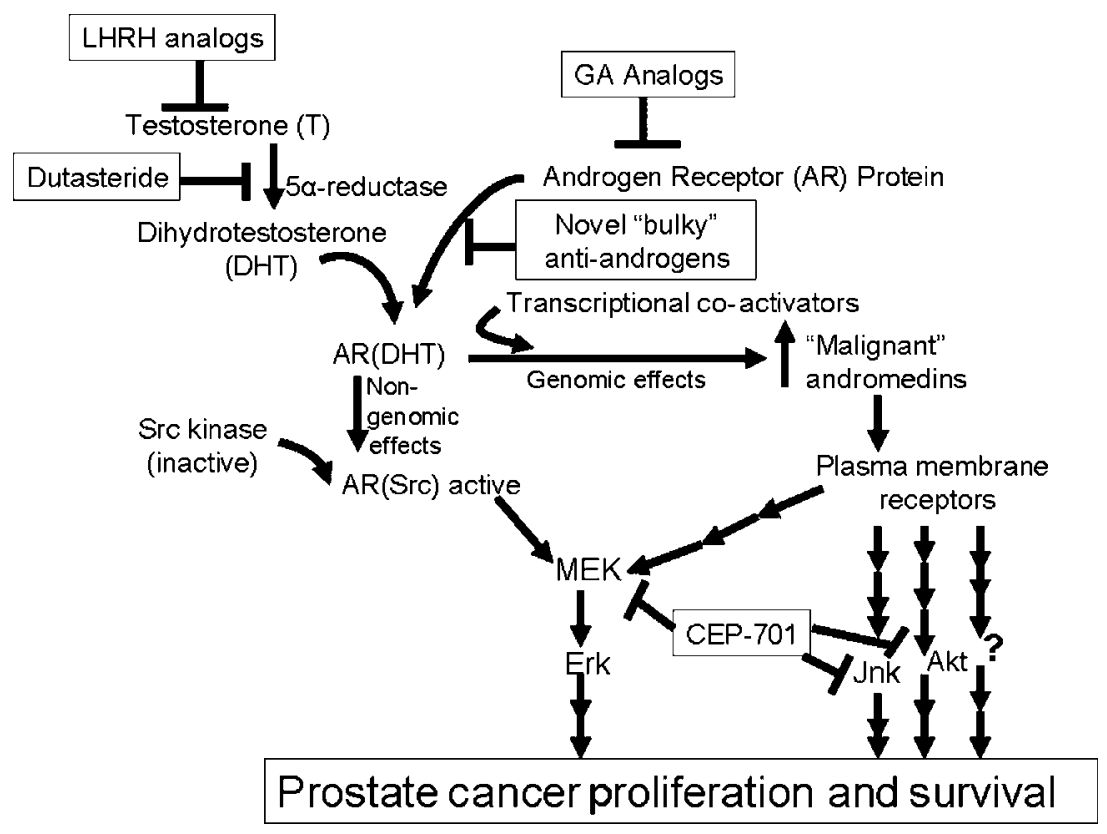

Figure 2 Overview of androgen receptor pathways in prostate cancer cells with the sites of action denoted for specific therapeutic small molecule inhibitors.

Presently, surgical or medical (i.e. LHRH analog/ antagonist) suppression of the major circulating androgen (i.e. testosterone) is the usual target of androgen ablation therapy. While this lowers the level of circulating testosterone via effects on the testes, it does not entirely eliminate testosterone and the biological impact of the low level of testosterone remaining can be amplified by the conversion of testosterone to DHT catalyzed by $5 \alpha$-steroid reductase (i.e. DHT is 10 times more potent than testosterone in transcriptional induction (Deslypere et al. 1992)). There are two distinct genes encoding $5 \alpha$-steroid reductase ( $5 \alpha$-SR) activity (i.e. type one and type two $5 \alpha$-SR) (Titus et al. 2005a). The type one enzyme is expressed by a variety of tissues, particularly skin fibroblasts and liver hepatocytes, while type two is more restrictively expressed by prostate and male accessory sex tissues, stromal and epithelial cells as well as liver hepatocytes (Titus et al. 2005a). Prostate cancer cells express modest levels of type two $5 \alpha$-SR, but have significant expression of the type one $5 \alpha-\mathrm{SR}$ (Titus et al. 2005a). Finasteride, Fig. 3, is a selective inhibitor of type two $5 \alpha$-SR and has been FDA approved for treatment of BPH. Likewise, dutasteride, which is a dual type one and two inhibitor, has been approved for BPH and is being tested presently for its chemoprevention ability for prostatic cancer development (Fig. 3).

Both type one and type two isozymes are expressed in prostatic cancer (Xu et al. 2006). We have also documented that daily treatment with finasteride reduces the level of DHT in both normal and malignant rodent and human prostatic tissues which have low, but not high, type one $5 \alpha$-SR (Lamb et al. 1992, Xu et al. 2006). Such DHT reduction induces significant regression of the normal sex accessory tissues (e.g. prostate and seminal vesicles) and growth of malignant prostate cancer with low type one $5 \alpha$-SR. In contrast, finasteride had no statistical effect upon the growth of a rodent prostate cancer, which expresses a high level of the type one isoform of $5 \alpha-\mathrm{SR}$ (e.g. Dunning H-tumor; Lamb et al. 1992, Xu et al. 2006). These results document that prostatic cancer cells can continue to grow at DHT levels which are unable to prevent the death of normal prostatic epithelial cells (i.e. prostatic cancer cells are hypersensitive to DHT
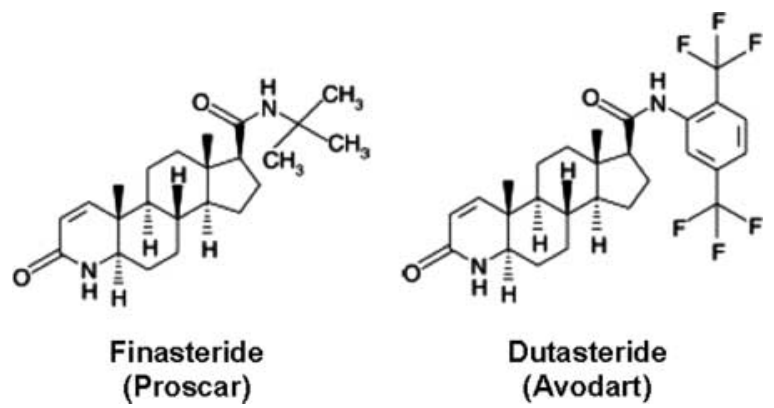

Figure 3 Chemical structures of finasteride and dutasteride. 
compared to normal tissue) (Ellis \& Isaacs 1985). In contrast to finasteride, daily treatment with dutasteride caused both growth inhibition of the Dunning H-tumor as well as regression of the normal sex accessory tissue (Xu et al. 2006). This dutasteride response was associated with a much greater reduction in DHT levels in both tissues than that induced by finasteride. In additional studies, it was documented that by combining oral daily dutasteride, but not finasteride, with androgen ablation, an additive inhibition of the growth of LNCaP human prostate cancer cells (i.e. cells which express a moderate level of type one $5 \alpha-\mathrm{SR}$ ) was produced in nude mice which is greater than that produced by castration alone (Xu et al. 2006). These results show that even after castration there is still a measurable level of androgen-induced malignant growth which can be suppressed by preventing the testosterone to DHT amplification via $5 \alpha$-reductase inhibition.

The importance of this realization is highlighted by two publications (Nishiyama et al. 2005, Titus et al. $2005 b)$ ). In the Nishiyama et al. paper, liquid chromatography-mass spectrometry (LC-MS) was used to analyze the tissue DHT levels in serum and prostatic biopsies from men with localized prostate cancer before and after androgen ablation therapy (i.e. induced surgically $(n=5)$ or by LHRH $(n=25))$. They documented that the pre-treatment tissue DHT levels $18.7 \pm 9.7 \mathrm{nM}$, which were reduced $75 \%$ to $4.6 \pm$ $4.5 \mathrm{nM}$ following 6 months of androgen ablation. Titus et al. also used LC-MS to show that in androgen ablation recurrent prostate cancer patients, DHT content in cancer tissue (i.e. $1.25 \mathrm{nM}$ ) was reduced $91 \%$ compared with androgen-stimulated BPH tissue $(13.7 \mathrm{nM})$ (Titus et al. 2005b). It is relevant to point out that the level of DHT in human prostate cancer growing in intact rats (i.e. 10-20 nM) is essentially identical to that of prostate cancers in humans (Ellis \& Isaacs 1985). It is also critical to point out that until DHT levels in human cancers were lowered to $\leq 5 \mathrm{nM}$ (i.e. similar to levels produced by androgen ablation in humans), no inhibition of human cancer growth occurred (Lamb et al. 1992). These combined results validate the use of a dual $5 \alpha$-reductase inhibitor, such as dutasteride in combination with LHRH analog to further lower DHT levels within prostate cancers. Presently, such LHRH/ dutasteride combinational therapy is being tested by GlaxoSmithKline in men with metastatic prostatic cancer who have a rising PSA level while on LHRH monotherapy. For these studies, serum PSA decreases are being used as an intermediate end point with survival being the ultimate objective criterion.

This further lowering of prostate cancer DHT when LHRH analog and dutasteride are combined is particularly relevant when coupled with the demonstration that a two- to five-fold increase in AR mRNA is the only gene expression change consistently associated with androgen ablation failure (Chen et al. 2004). This results in a two- to three-fold increase in the levels of AR protein with androgen ablationresistant prostatic cancer cells (Chen et al. 2004). These observations have led to the suggestion that nonsteroidal anti-androgen AR antagonistic-like casodex should be combined with LHRH to produce a more 'complete androgen blockage.' Unfortunately, addition of anti-androgen with LHRH has added little to survival of prostate cancer patients (Prostate Cancer Trialists' Collaborative Group 1995, Eisenberger et al. 1998). An explanation for this limited effect is provided by the demonstration that while antiandrogen antagonists bind to the ligand-binding domain (LBD) of AR preventing DHT binding, such AR anti-androgen antagonist complexes can be structurally 'forced' into an agonist conformation by binding with other proteins to allow transcriptional coactivation like p160 and p300, which are overexpressed in androgen ablation-resistant prostate cancer to bind and activate transcription of 'malignant' andromedin genes. These results suggest two possible methods preventing such malignant signaling. The first is to develop therapies which cause the downregulation of the AR protein. Indeed, this possibility has been documented in vitro using AR-positive human prostate cancer cell lines (Chen et al. 1998, Eder et al. 2002, Solit et al. 2002, Zegarra-Moro et al. 2002, Chen et al. 2004, Liao et al. 2005, Yang et al. 2005). Several approaches are currently available to lower AR. These include heat shock protein-90 inhibitors (Solit et al. 2002), RNA interference (Liao et al. 2005, Yang et al. 2005) and ribozyme (Zegarra-Moro et al. 2002), and antisense (Eder et al. 2002, Zegarra-Moro et al. 2002). A logical prediction emerging from these studies is that reducing AR expression to a critical level would not only slow the growth of prostate cancer cells, but would also result in apoptosis. Two recent papers as well as our own unpublished data have shown that, indeed, if the levels of AR are lowered sufficiently, prostate cancer cells die by apoptosis (Liao et al. 2005, Yang et al. 2005).

Along these lines, the chaperone ability of the $90 \mathrm{kDa}$ heat shock protein (HSP-90) for AR has become a logical target. HSP-90 binds to a variety of intracellular proteins, including the ligand-unoccupied AR. Upon binding of androgen to the AR complexed with HSP-90, there is an ATP-driven cycle that involves ARP binding to an N-terminal pocket in HSP-90 followed by subsequent hydrolysis to ADP and release of the 
androgen-occupied AR from the HSP-90 complex (Young \& Hartl 2000). Geldanamycin (GA) is a benzoquinone ansamycin antibiotic that binds as a competitive ATP analog to the N-terminal ATP binding pocket of HSP-90. This binding of GA prevents the release of AR from its HSP-90 complex and results in the ubiquitinization and subsequent degradation of the AR but not HSP-90 protein (Segnitz \& Gehring 1997, Kuduk et al. 2000). Such GA-induced AR downregulation results in the apoptosis of $\mathrm{LNCaP}$ prostate cancer cells (Solit et al. 2002). This observation was confirmed using a panel of androgen-resistant human prostatic cancer cell lines, some of which have: (1) a single point mutation in the $\mathrm{LBD}$ region of their $\mathrm{AR}$ (i.e. $\mathrm{LNCaP}$ ), or (2) several point mutations in the LBD region of the AR (i.e. MDA-PC-2B), or (3) a point mutation in the LBD region and an internal duplication of exon 3 in its AR producing a larger AR protein that is prone to proteolytic degradation producing a constitutively active AR composed of its N-terminal, DNA binding and hinge region but no LBD (i.e. CWR22Rv1) and (4) wild-type AR (i.e. LAPC-4) (van Bokhoven et al. 2003). All of these human prostate cancer cell lines have comparable levels of both AR and HSP-90 (Fig. 4, left panel) and all produce downregulation of their AR protein following GA treatment (Fig. 4, right panel). (Note: the number under the lane for each cell line is the normalized value for AR and HSP-90, in Fig. 4, left panel, compared with the respective amount detected in LNCaP cells or normalized vs control (i.e. untreated cells, in Fig. 4, right panel)). These normalized values show that steady state AR expression is consistently more than 10-fold higher in androgen ablation-resistant prostate cancer cell lines as compared with normal human prostatic stromal cells such as $4 \mathrm{~S}$ and $6 \mathrm{~S}$ (AR western blots for these lines exposed 100 times larger than the cancer cells). In contrast, HSP-90 levels are comparable between the cancer lines and normal prostatic stromal cells (Fig. 4, left panel). The concentration of GA which inhibits the growth of these HSP-90 expressing cancer cells by $50 \%$ (i.e. $\mathrm{IC}_{50}$ value) ranges from a low of $15 \pm 2 \mathrm{nM}$ for LAPC-4 to a high of $65 \pm 10 \mathrm{nM}$ for CWR22Rv1 cells, which is identical to the concentration of GA needed to reduce AR protein levels by more than $80 \%$ within 24 hours of treatment (Fig. 4, left panel). Unfortunately, GA undergoes hepatic metabolism and is too toxic and insoluble for systemic delivery. Therefore, GA analogs are being developed for clinical testing. These include 17-(allylamino)-17-demethoxygeldanamycin (17AAG) and its more hydrophilic derivative 17-(dimethyl-aminoethylamino)-17-demethoxygeldanamycin (17-DMAG) (Glaze et al. 2005).

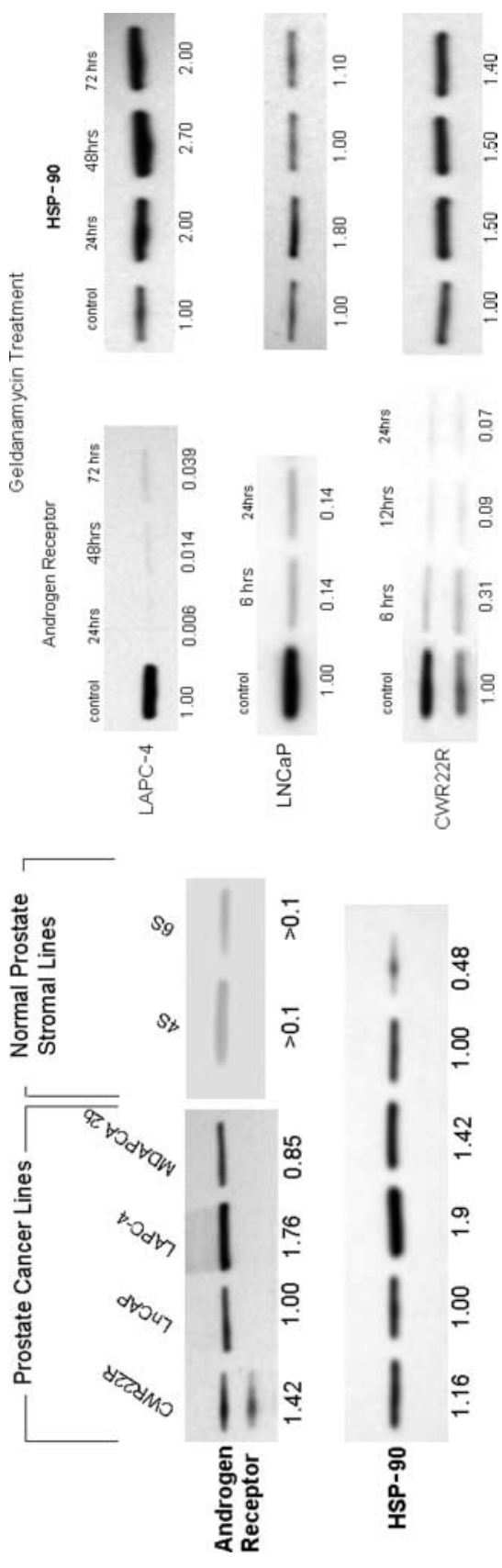

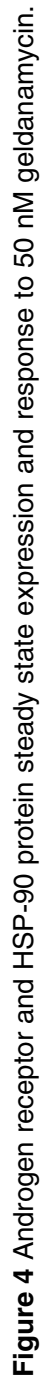


A second and complementary approach to block AR signaling in the low androgen environment following androgen ablation is to develop better small molecule anti-androgen antagonistic configuration, which can prevent the AR from being 'forced' into an agonistic configuration. Development of such new anti-androgens should be possible based upon the growing understanding of the structural biology of the AR. The $\mathrm{AR}$ is a member of the steroid/nuclear receptor super family of ligand-dependent transcription factors. AR contains a central DNA binding domain, which separates the receptor amino $(\mathrm{N})$ terminus from the carboxy (C) terminus. The $\mathrm{N}$ terminus contains an activation function (AF-1) transactivation domain and the $\mathrm{C}$ terminus harbors the LBD and the liganddependent activator function transcriptional (AF-2) domain. Previous studies have demonstrated that an $\mathrm{N}$ to $\mathrm{C}$ terminal intra molecular interaction of $\mathrm{AR}$ monomers activates functional transcription via their DNA binding and dimerization (Gregory et al. 2001, He et al. 2004, Bai et al. 2005). Co-activators, such as the p160 family of co-activators like SRC-1, transcriptional intermediary factor-2 (TIF-2), or glucocorticoid receptor-interacting protein 1 were originally defined as factors that increase the total amount of induced gene product with saturating concentrations of hormone. As will be discussed, X-ray crystallographic studies indicate that AR can adopt a structural fold involving helices 3, 4, 5 and 12 of the LBD (i.e. AF-2 domain) with either an agonist conformation which binds such co-activator proteins or an antagonist conformation which binds co-repressors (He et al. 2004, Bai et al. 2005, Bohl et al. 2005). The nuclear receptor co-repressor $(\mathrm{NCoR})$ and the related silencing mediator for retinoid and thyroid hormone receptors (SMRT) were initially discovered on the basis of their ability to bind to ligand-free nuclear receptors, including AR, preventing them from inducing transcription. Such co-repressors also interact with antagonist-bound AR to prevent transcription.

The AR has been crystallized in the presence of both its natural ligand DHT and the anti-androgen casodex (i.e. bicalutamide). These analyses raise the issue that casodex is not structurally 'bulky' enough to "lock" the AR in an antagonistic conformation (Bohl et al. 2005). Structural analyses suggest that when such a less bulky antagonist binds to the ligand-unoccupied AR, it induces conformational change which, while not producing a full agonist state, makes it easier for the partially activated AR to complete such an agonist conformation, particularly if there is overexpression of co-activators. Indeed, this possibility has been suggested as the mechanism for the anti-androgen withdrawal response occurring in patients and the conversion of anti-androgens like casodex to full agonist activity in a low androgen environment following experimental upregulation of co-activators in vitro (Isaacs \& Isaacs 2004). The specific mechanism or such a forced conformation is unknown but the critical role for co-activator displacement of corepressor binding for activating AR sensitive gene transcription has been documented. Androgen ablation-resistant prostate cancer overexpresses p160 and p300 (e.g. CREB-binding protein (CBP) co-activator protein (Gregory et al. 2001, 2004, Debes et al. 2002, Culig et al. 2004). Phosphorylation of these overexpressed co-activators induced by cross-talk with other signaling cascades presumably allows these phosphorylated forms to bind to the AF-2 domain, displacing co-repressors, and forcing the AR into an agonist state either without ligand or when bound by low molecular weight antagonists (Gregory et al. 2001, Chen et al. 2004, Estebanez-Perpina et al. 2005, Hodgson et al. 2005). The working hypothesis is that AR conformation when either unoccupied by agonist ligands or bound by low molecular weight partial agonists or antagonists can be forced by the binding of co-regulators to displace co-repressors and undergo change to a full agonist conformation of the AF-2 domain of the AR. Therefore, a novel strategy to block gene activation by AR in such androgen ablationfailing patients is to develop 'bulky' anti-androgens which bind to LBD and structurally lock the AF-2 domain of the AR surface in an antagonist conformation thus not allowing its AF-2 domain to be forced into the agonist state.

Therefore, we have used structural biology to design small molecule drugs, which can lock the C-terminal half of the AR in an antagonist form. An initial approach we are taking is based upon the recent structural biology studies published by the Fletterick laboratory which have documented that the binding of agonist to the ligand pocket, within the LBD portion of the AR (Fig. 5A and B), induces the correct conformation of an agonistic groove (i.e. AF-2 domain) involving helices 3, 4, 5 and 12 (Estebanez-Perpina et al. 2005). Once induced to form, this agonistic groove does not bind corepressors (e.g. NCoR) but instead binds co-activators (e.g. Src1 and Src2) to initiate the agonist function of the AR (Fig. 5C). In contrast, Hodgson et al. (2005, Fig. 5C) have shown that when ligands such as mifepristone (i.e. RU-486) bind the ligand pocket of the LBD, the AF-2 position of the receptor is converted into an antagonistic form which induces not co-activator but NCOR-binding antagonizing AR function, (Hodgson et al. 2005, Fig. 6). This is because mifepristone has a bulky 

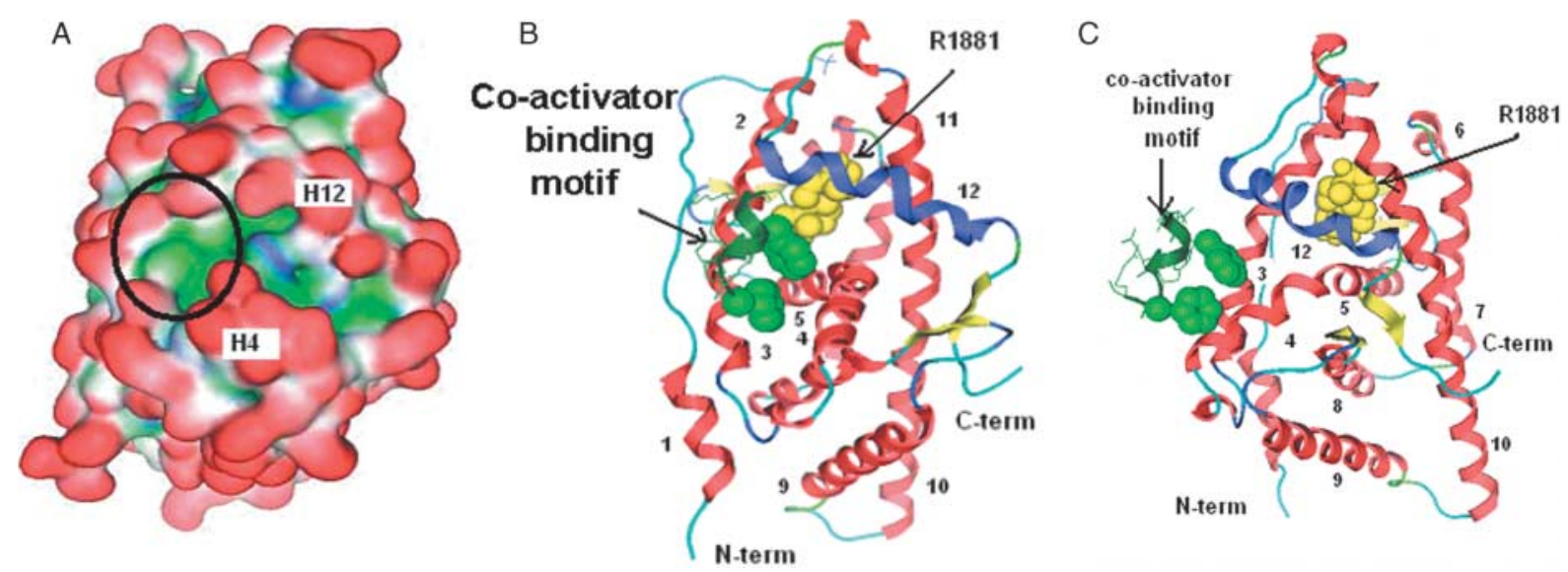

Figure $5(A)$ Space filling diagram of the AR LBD with bound R1881. Locations of helices 12 and 4 are marked in the image. AF-2 co-activator binding groove is located in the area within the circle. (B) Ribbon diagram of AR-LBD shown with a co-activator binding motif (in green) bound in the AF-2 binding groove in its agonist configuration. Bound R1881 ligand is shown in yellow. (C) Same as B only rotated $90^{\circ}$. C-term, Carboxy terminus; N-term, amino terminus.

side-chain addition (i.e. dimethylaniline) at the $11 \beta$ position of the C-ring steroid nucleus (Fig. 7). This bulky substitution at the $11 \beta$ position of mifepristone displaces activation helix 12 of the AR, progesterone, and glucocorticoid receptors hindering their agonist conformation (Kauppi et al. 2003).

It is this displacement that allows mifepristone to be an anti-androgen as well as an anti-progestin and antiglucocorticoid. As a therapeutic agent, mifepristone, however, has two disadvantages. First, it has unwanted anti-glucocorticoid activity. Second, structural biology modeling has revealed that while mifepristone does displace helix 12 of the AR, such displacement is modest, raising the possibility that binding of coactivators to the AF-2 domain, defined as the 3, 4, 5, 12 groove of the AR-LBD, could reposition helix 12 back into an agonist conformation. Thus, a better mifepristone-like anti-androgen can be designed based upon having bulkier and stiffer side chains at the $11 \beta$ position of the C-ring of the steroid nucleus (Fig. 7). Along this line, studies by Muddana et al. (2004) have demonstrated that instead of using the mifepristone as the core steroid backbone, $\Delta^{9}$-19-nortestosterone can be used as the core backbone for such $11 \beta$ position analogs and
A

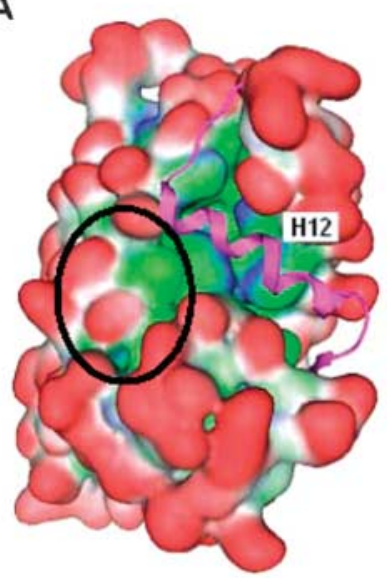

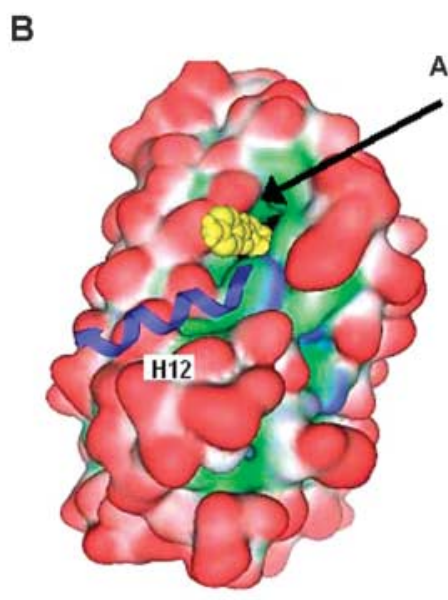

New

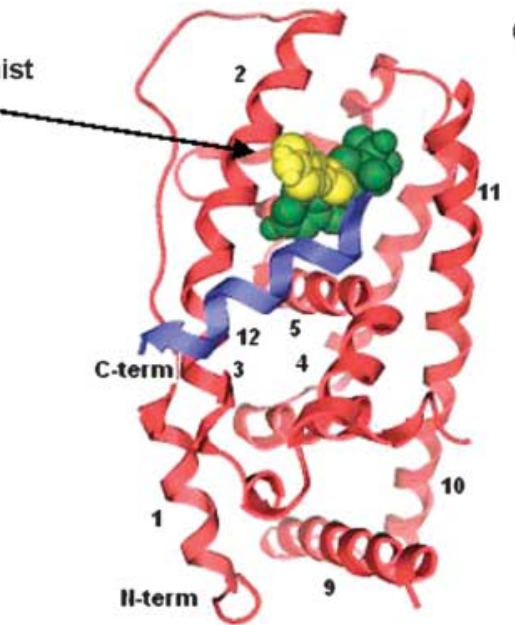

C

Figure $6(A)$ Space filling diagram of AR-LBD with helix $(\mathrm{H}) 12$ in the agonist conformation of the AF-2 groove denoted as a purple ribbon. (B) Helix 12 in the antagonist conformation induced by its displacement by the stiff side chain at position 11 of newly designed analog. (C) Ribbon diagram with steroid analog core in green and stiff side chain in yellow. 
thereby eliminate the anti-glucocorticoid activity of the derivatives. Thus, we have used the available structural biology data to design $\Delta^{9}$-19-nortestosterone analogs, which should have high (i.e. pM) affinity for the ligandbinding pocket of the AR but not the glucocorticoid receptor, and which have a bulky and stiff side chain composed of repeating numbers (i.e. $N=1-3$ ) of paraamino-benzoate units at the $11 \beta$ position (Fig. 7). These new analogs should be able to bind to the ligand pocket of the LDB of AR and tether the stiff 11 position side chain to sterically prevent helix 12's rotation to generate the co-activator binding agonist groove formed by helices 3, 4, 5 and 12 (Fig. 6).

The advantage of a combinatorial approach to blocking the AR signaling cascade in androgen ablation-failing prostatic cancer patients is that no one point in the cascade has to be completely inhibited if several complementary steps are significantly downregulated. For example, if the levels of ligand and AR receptor are lowered but not entirely eliminated, a reduced amount of genomic (i.e. transcriptional) and non-genomic signaling could still occur. In fact, under this low ligand, low AR situation, the major portion of the AR protein is located within the cytoplasm. This situation is favorable to AR binding to cytoplasmic signaling complexes. For example, Unni et al. (2004) have demonstrated that in androgen ablation-resistant human prostatic cancer cells, Src kinase protein binds to a cytoplasmic scaffolding protein known as MNAR. In this complex, Src's kinase activity is not active. AR can form tertiary complexes with MNAR/Src via its proline repeat region in its $\mathrm{N}$-terminal region, resulting in activation of the Src kinase. This initiates a signaling cascade involving Src kinase phosphorylating the MAPK kinase, Mek. Mek then phosphorylates and activates Erk-MAPK, which produces a signaling cascade stimulating the proliferation and survival of prostatic cancer cells (Mellinghoff et al. 2004, Unni et al. 2004). We have documented the importance of this non-genomic signaling pathway by the demonstration that inhibition of Mek's kinase activity by the small molecule inhibitor UO126 profoundly inhibits the in vitro proliferation, but does not induce the death of AR-positive, but less seen AR-negative cells (Uzgare \& Isaacs 2004). In additional published studies, we have provided further support for the importance of these nongenomic effects of AR by the demonstration that there is a dissociation between AR responsiveness for malignant growth vs transcriptional (i.e. genomic) regulation of prostate-specific differentiation markers PSA, hK2 and PSMA in human androgen ablation-resistant prostate cancer cell lines (Denmeade et al. 2003).

These results are consistent with non-genomic signaling effects being critically involved in the AR regulation of prostate cancer cell growth. To address these non-genomic effects, the causal role of the activation of the downstream Akt and MAP kinases associated with development and progression of prostate cancer to the lethal androgen ablation-resistant state was studied. Using UO126, SB203580, SP600125, and (AIN small molecule inhibitors of Mek, p38 MAPK, Jnk MAPK, and Akt kinase respectively) it was documented that inhibition of either MEK or Jnk results in apoptosis of AR-negative

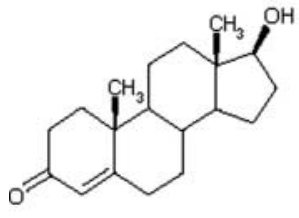

Testosterone

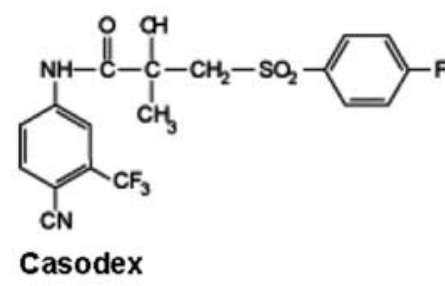

Casodex

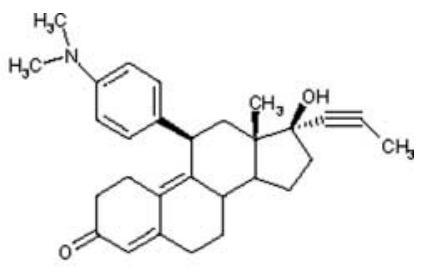

Mifepristerone

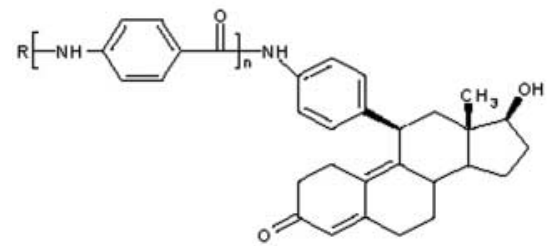

11b-ßenzyl-D9-19-nortestosterone analog

Figure 7 Chemical structures of compounds discussed in the text. 
normal prostatic TA cells, but such apoptosis of androgen ablation-resistant prostate cancer cells required simultaneous inhibition of Mek, Jnk and Akt (Uzgare \& Isaacs 2004). These results demonstrate that prostate cancer progression to a lethal androgen ablation-resistant state involves the acquisition of an enhanced redundancy in downstream survival signaling.

Cephalon, Inc. (Frazer, PA, USA), has developed a large library of indolocarbazole kinase inhibitors as possible therapeutics for neurological disease and cancer. From these studies, CEP-701 (Fig. 8), a potent $\mathrm{nM}$ inhibitor of kinase activated by binding of neurotrophins (i.e. NGF, BDNF, NT-3) to their cognate receptors (i.e. TrkA for NGF, TrkB for BDNF, and TrkC for NT-3) was discovered to be an effective inhibitor of both rodent and human prostate cancer cells in vitro and in vivo (George et al. 1999, Weeraratna et al. 2000, 2001). CEP-701's anti-cancer efficacy involves inhibiting the autocrine-signaling pathway used by prostate cancer cells to stimulate their growth (i.e. prostate cancer cells produce several types of neurotrophins and express their appropriate receptors (Weeraratna et al. 2000)). These pre-clinical studies led to CEP-701 being developed for clinical testing (Marshall et al. 2005).

Remarkably, we have shown that treatment of these same androgen ablation-resistant human prostate cancer cells with CEP-701 at a 50-100 times lower concentration (i.e. $200 \mathrm{~nm}$ ) produces better inhibition of in vitro growth of these cancer cells than monotherapy with $20 \mu \mathrm{M} \mathrm{p} 38,10 \mu \mathrm{M}$ Mek, $20 \mu \mathrm{M}$ Jnk, or $20 \mu \mathrm{M}$ Akt inhibitors (Weeraratna et al. 2000, Uzgare \& Isaacs 2004). Even more remarkable, CEP-701 monotherapy produces essentially an identical inhibitory response induced by combining Mek, Jnk, and Akt inhibition (Weeraratna et al. 2000, Uzgare \& Isaacs 2004). We have now documented that the mechanism for the

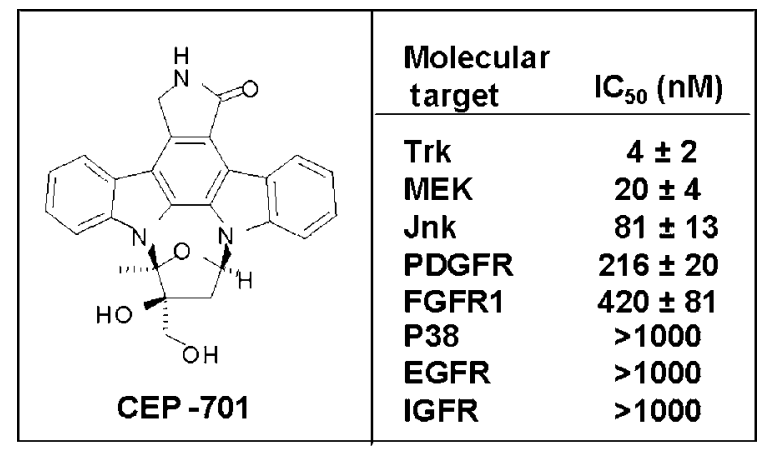

Figure 8 Chemical structure of CEP-701 and IC ${ }_{50}$ for CEP-701 inhibition of selected purified kinases.

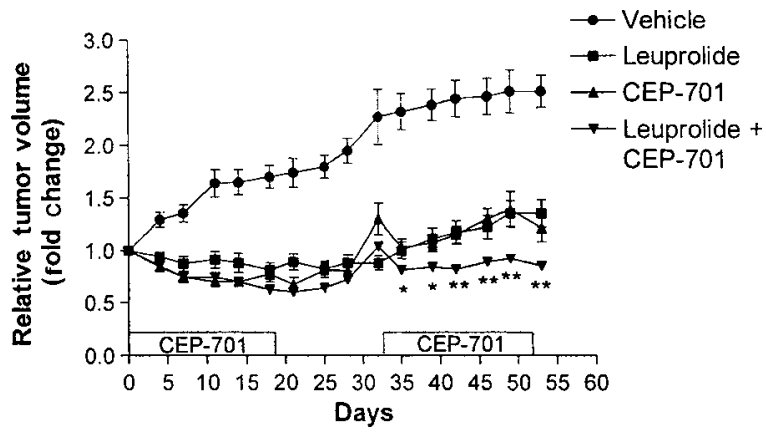

Figure 9 Effects of CEP-701, leuprolide alone or the simultaneous combination on the growth of Dunning $\mathrm{H}$ prostate cancer in rats. * and ${ }^{* *}$ indicates $P<0.01$ compared with and either leuprolide or CEP-701 monotherapy treatment groups.

outstanding anti-prostatic cancer efficacy of CEP-701 monotherapy is due to its potent (i.e. nM) ability to inhibit Mek and Jnk directly (Fig. 8).

Based on this ability, we would predict that combining CEP-701 to suppress the non-genomic AR signaling would enhance the efficacy of androgen ablation targeted at suppression of AR's genomic (i.e. transcriptional) effects. To test whether such enhanced efficacy is produced by combining CEP-701 with androgen ablation, the previously described Dunning R-3327 H rat prostatic cancer model was used. H-tumor-bearing rats were treated with either: (1) vehicle (i.e. intact controls); (2) LHRH analog continuously via SQ depot (i.e. leuprolide at a dose of $5.2 \mathrm{mg} / \mathrm{kg}$ every three weeks which lowers serum testosterone levels to $<0.1 \mathrm{ng} / \mathrm{ml}$ ); (3) $10 \mathrm{mg} / \mathrm{kg}$ of CEP-701 orally twice a day for two 21 day cycles (separated by 10 days off-drug); or 4) LHRH analog depot plus CEP-701 for two cycles. The effects of these treatments on tumor volumes are presented in Fig. 9. These results show that CEP-701 does enhance the efficacy of LHRH when given simultaneously; however, no animal was cured by such a combinatorial therapy (George et al. 1999).

\section{Conclusions}

As summarized in Fig. 2, there are multiple points in the AR signaling pathway that can be approved as therapeutic targets for this CART therapy. These include LHRH analogs, dual $5 \alpha$-reductase inhibitors, kinase inhibitors, and bulky anti-androgens. Presently, we are synthesizing and testing bulky steroidal anti-androgens for inclusion in this CART therapy. Such optimal CART therapy could be developed clinically by phase II evaluation in metastatic prostate cancer patients who have a rising 
PSA while on LHRH monotherapy or LHRH plus casodex. As an intermediate end point, the serum PSA response (e.g. decrease in serum PSA rise with time) could be used as proposed by D'Amico et al. (2004) in such phase II trials to document whether addition of the specific agent to metastatic patients progressing on standard LHRH analog treatment with a rising PSA, produces a PSA response. If it does, dose-finding studies could define the lowest dose of the additional agent that produces such PSA responses. Using this approach, optimal CART therapy could be developed in a stepwise fashion to define the fewest number of agents and the lowest doses needed to produce the best PSA response in progressing patients. Once defined, this optimal CART therapy would be tested in phase III trials.

\section{Funding}

Support for this research was provided by NIH Grant R01DK52645 and by Sponsored Research Agreements with Cephalon, Inc. and GlaxoSmithKline. Dr Isaacs is a member of the Scientific Advisory Board of Cephalon, Inc. and a consultant for GlaxoSmithKline for which he receives monetary remuneration. He has received sponsored research support from both Cephalon, Inc. and GlaxoSmithKline. Dr Denmeade is a consultant to Cephalon, Inc.

Drs Isaacs' and Denmeade's financial interest and research relationship with Cephalon, Inc. and GlaxoSmithKline have been disclosed to the Johns Hopkins School of Medicine Conflict of Interest Committee and are being managed accordingly.

\section{References}

Bai S, He B \& Wilson EM 2005 Melanoma antigen gene protein MAGE-11 regulates androgen receptor function by modulating the interdomain interaction. Molecular Cell Biology 25 1238-1257.

Bohl CE, Gao W, Miller DD, Bell CE \& Dalton JT 2005 Structural basis for antagonism and resistance of bicalutamide in prostate cancer. PNAS 102 6201-6206.

Castoria G, Lombardi M, Barone MV, Bilancio A, Di Domenico M, De Falco A, Verricchio L, Bottero D, Nanayakkara M, Migliaccio A \& Auricchio F 2004 Rapid signaling pathway activation by androgens in epithelial and stromal cells. Steroids 69 517-522.

Chen S, Song CS, Lavrovsky Y, Bi B, Vellanoweth R, Chatterjee B \& Roy AK 1998 Catalytic cleavage of the androgen receptor messenger RNA and functional inhibition of androgen receptor activity by a hammerhead ribozyme. Molecular Endocrinology 12 1558-1566.
Chen CD, Welsbie DS, Tran C, Baek SH, Chen R, Vessella R, Rosenfeld MG \& Sawyers CL 2004 Molecular determinants of resistance to antiandrogen therapy. Nature Medicine 10 26-27.

Culig Z, Comuzzi B, Steiner H, Bartsch G \& Hobisch A 2004 Expression and function of androgen receptor coactivators in prostate cancer. Journal of Steroid Biochemistsry and Molecular Biology 92 265-271.

D'Amico AV, Moul JW, Carroll PR, Cote K, Sun L, Lubeck D, Renshaw AA, Loffredo M \& Chen MH 2004 Intermediate end point for prostate cancer-specific mortality following salvage hormonal therapy for prostate-specific antigen failure. Journal of the National Cancer Institute 96 509-515.

De Marzo AM, Meeker AK, Epstein JI \& Coffey DS 1998 Prostate stem cell compartments: expression of the cell cycle inhibitor p27Kip1 in normal, hyperplastic, and neoplastic cells. American Journal of Pathology 153 911-919.

Debes JD, Schmidt LJ, Huang H \& Tindall DJ 2002 P300 mediates androgen-independent transactivation of the androgen receptor by interleukin 6. Cancer Research 62 5632-5636.

Denmeade SR, Sokoll LJ, Dalrymple S, Rosem DM, Gady AM, Bruzek D, Ricklis RM \& Isaacs JT 2003 Dissociation between androgen responsiveness for malignant growth vs expression of prostate specific differentiation markers PSA, hK2, and PSMA in human prostate cancer models. The Prostate 54 249-257.

Deslypere JP, Young M, Wilson JD \& McPhaul MJ 1992 Testosterone and 5-alpha dihydrotestosterone interact differently with the androgen receptor to enhance transcription of the MMTV-CAT reporter gene. Molecular and Cellular Endocrinology 88 15-22.

Eder IE, Hoffmann J, Rogatsch H, Schafer G, Zopf D, Bartsch G \& Klocker H 2002 Inhibition of LNCaP prostate tumor growth in vivo by an antisense oligonucleotide directed against the human androgen receptor. Cancer Gene Therapy 9 117-125.

Eisenberger MA, Blumenstein BA, Crawford ED, Miller G, McLeod DG, Loehrer PJ, Wilding G, Sears K, Culkin DJ, Thompson IM Jr et al. 1998 Bilateral orchiectomy with or without flutamide for the treatment of patients with metastatic prostate cancer. New England Journal of Medicine 339 1036-1042.

Ellis WJ \& Isaacs JT 1985 Effectiveness of complete versus partial androgen withdrawal therapy for the treatment of prostatic cancer as studied in the Dunning R-3327 system of rat prostatic adenocarcinomas. Cancer Research $\mathbf{4 5}$ 6041-6050.

Estebanez-Perpina E, Moore JM, Mar E, Delgado-Rodrigues E, Nguyen P, Baxter JD, Buehrer BM, Webb P, Fletternick RJ \& Guy RK 2005 The molecular mechanisms of coactivator utilization in ligand-dependent transactivation by the androgen receptor. Journal of Biological Chemistry 280 8060-8068. 
Gao J \& Isaacs JT 1998 Development of an androgen receptor null model for identifying the site of initiation for androgen stimulation of proliferation and suppression of programmed (apoptotic) death of PC-82 human prostate cancer cells. Cancer Research 58 3299-3306.

Gao J, Arnold JT \& Isaacs JT 2001 Conversion from a paracrine to an autocrine mechanism of androgenstimulated growth during malignant transformation of prostatic epithelial cells. Cancer Research 61 5038-5044.

Garraway LA, Lin D, Signoretti S, Waltregny D, Dilks J, Bhattacharya N \& Loda M 2003 Intermediate basal cells of the prostate: in vitro and in vivo characterization. Prostate 55 206-218.

George DJ, Dionne CA, Jani J, Angeles T, Murakata C, Lamb J \& Isaacs JT 1999 Sustained in vivo regression of Dunning $\mathrm{H}$ rat prostate cancers treated with combinations of androgen ablation and Trk tyrosine kinase inhibitors, CEP-751 (KT-6587) or CEP-701 (KT-5555). Cancer Research 59 2395-2401.

Glaze ER, Lambert AL, Smith AC, Page JG, Johnson WD, McCormick DL, Brown AP, Levine BS, Covey JM, Egorin MJ, Eiseman JL, Holleran JL, Sausville EA \& Tomaszewski JE 2005 Preclinical toxicity of a geldanamycin analog, 17-(dimethylaminoethylamino)-17demethoxygeldanamycin (17-DMAG), in rats and dogs: potential clinical relevance. Cancer Chemotherapy and Pharmacology 56 637-647.

Gregory CW, He B, Johnson RT, Ford OH, Mohler JL, French FS \& Wilson EM 2001 A mechanism for androgen receptor-mediated prostate cancer recurrence after androgen deprivation therapy. Cancer Research 61 4315-4319.

Gregory CW, Fei X, Ponguta LA, He B, Bill HM, French FS \& Wilson EM 2004 Epidermal growth factor increases coactivation of the androgen receptor in recurrent prostate cancer. Journal of Biological Chemistry 279 7119-7130.

He B, Gampe RT Jr, Kole AJ, Hnat AT, Stanley TB, An G, Stewart EL, Kalman RI, Minges JT \& Wilson EM 2004 Structural basis for androgen receptor interdomain and coactivator interactions suggests a transition in nuclear receptor activation function dominance. Molecular Cell 16 425-438.

Hodgson MC, Astapova I, Cheng S, Lee LJ, Verhoeven MC, Choi E, Balk SP \& Hollenberg AN 2005 The androgen receptor recruits nuclear receptor CoRepressor (N-CoR) in the presence of mifepristone via its $\mathrm{N}$ and $\mathrm{C}$ termini revealing a novel molecular mechanism for androgen receptor antagonists. Journal of Biological Chemistry $\mathbf{2 8 0}$ 6511-6519.

Isaacs JT 1987 Control of cell proliferation and cell death in the normal and neoplastic prostate: a stem cell model. In Benign Prostatic Hyperplasia, Report no. NIH 87-2881, pp. 85-94. Eds CH Rogers, DS Coffey, G Cunha, JT Grayhack, F Hinman Jr \& R Horton. Department of Health and Human Services, Bethesda: National Institutes of Health.

Isaacs JT \& Isaacs WB 2004 Androgen receptor outwits prostate cancer drugs. Nature Medicine 10 26-27.
Kauppi B, Jakob C, Farnegardh M, Yang J, Ahola H, Alarcon M, Calles K, Engstrom O, Harlan J, Muchmore S, Ramqvist AK, Thorell S, Ohman L, Greer J, Gustafsson JA, Carlstedt-Duke J \& Carlquist M 2003 The threedimensional structures of antagonistic and agonistic forms of the glucocorticoid receptor ligand-binding domain: RU-486 induces a transconformation that leads to active antagonism. Journal of Biological Chemistry 278 22748-22754.

Kuduk SD, Harris TC, Zheng FF, Sepp-Lorenzino L, Ouerfelli Q, Rosen N \& Danishefsky SJ 2000 Synthesis and evaluation of geldanamycin-testosterone hybrids. Bioorganic and Medicinal Chemistry Letters 10 1303-1306.

Kurita T, Wang YZ, Donjacour AA, Zhao C, Lydon JP, O'Malley BW, Isaacs JT, Dahiya R \& Cunha GR 2001 Paracrine regulation of apoptosis by steroid hormones in the male and female reproductive system. Cell Death and Differentiation 8 192-200.

Lamb JC, Levy MA, Johnson RK \& Isaacs JT 1992 Response of rat and human prostatic cancers to the novel 5-alpha-reductase inhibitor, SK\&F 105657. Prostate 21 15-34.

Liao X, Tang S, Thrasher JB, Griebling TL \& Li B 2005 Small-interfering RNA-induced androgen receptor silencing leads to apoptotic cell death in prostate cancer. Molecular Cancer Therapeutics 4 505-515.

Litvinov IV, De Marzo AM \& Isaacs JT 2003 Is the Achilles' heel for prostate cancer therapy a gain of function in androgen receptor signaling? Journal of Clinical Endocrinology and Metabolism 88 2972-2982.

Lu W, Luo Y, Kan M \& McKeehan WL 1999 Fibroblast growth factor-10. A second candidate stromal to epithelial cell andromedins in prostate. Journal of Biological Chemistry 274 12827-12834.

Marshall JL, Kindler H, Deeken J, Bhargava P, Vogelzang NJ, Rizvi N, Luhtala T, Boylan S, Dordal M, Robertson P, Hawkins MJ \& Ratain MJ 2005 Phase I trial of orally administered CEP-701, a novel neurotrophin receptorlinked tyrosine kinase inhibitor. Investigational New Drugs 23 31-37.

Mellinghoff IK, Vivanco I, Kwon A, Tran C, Wongvipat J \& Sawyers CL 2004 HER2/neukinase-dependent modulation of androgen receptor function through effects on DNA binding and stability. Cancer Cell 6 517-527.

Mohler JL, Gregory CW, Ford OH III, Kim D, Weaver CM, Petrusz P, Wilson EM \& French FS 2004 The androgen axis in recurrent prostate cancer. Clinical Cancer Research 10 440-448.

Muddana SS, Price AM, MacBride MM \& Peterson BR 2004 11-beta-alkyl-delta9-19-nortestosterone derivatives: highaffinity ligands and potent partial agonists of the androgen receptor. Journal of Medicinal Chemistry 47 4985-4988.

Nakano K, Fukabori Y, Itoh N, Lu W, Kan M, McKeehan WL \& Yamanaka H 1999 Androgen-stimulated human prostate epithelial growth mediated by stromal-derived fibroblast growth factor-10. Endocrinology Journal 46 405-413. 
Nishiyama T, Ishizaki F, Anraku T, Shimura H \& Takashashi K 2005 The influence of androgen deprivation therapy on metabolism in patients with prostate cancer. Journal of Clinical Endocrinology and Metabolism 90 657-660.

Nonomura N, Nakamura N, Uchida N, Noguchi S, Sato B, Sonoda T \& Matsumoto K 1988 Growth-stimulatory effect of androgen-induced autocrine growth factor(s) secreted from Shionogi carcinoma 115 cells on androgenunresponsive cancer cells in a paracrine mechanism. Cancer Research 48 4904-4908.

Ohlson N, Bergh A, Stattin P \& Wikstrom P 2006 Castrationinduced epithelial cell death in human prostate tissue is related to locally reduced IGF-1 levels. The Prostate (In press).

Prostate Cancer Trialists' Collaborative Group 1995 Maximum androgen blockade in advanced prostate cancer: an overview of 22 randomized trials with 3283 deaths in 5710 patients. Lancet 346 265-269.

Redding TW, Schally AV, Radulovic S, Milovanovic S, Szepeshazi K \& Isaacs JT 1992 Sustained release formulations of luteinizing hormone-releasing hormone antagonist SB-75 inhibit proliferation and enhance apoptotic cell death of human prostate carcinoma (PC-82) in male nude mice. Cancer Research 52 2538-2544.

Richardson GD, Robson CN, Lang SH, Neal DE, Maitland NJ \& Collins AT 2004 CD133, a novel marker for human prostatic epithelial stem cells. Journal of Cell Science 117 3539-3545.

Segnitz B \& Gehring U 1997 The function of steroid hormone receptors is inhibited by the Hsp90-specific compound geldanamycin. Journal of Biological Chemistry 272 18694-18701.

Shah RB, Mehra R, Chinnaiyan AM, Shen R, Ghosh D, Zhou M, Macvicar GR, Varambally S, Harwood J, Bismar TA, Kim R, Rubin MA \& Pienta KJ 2004 Androgenindependent prostate cancer is a heterogeneous group of disease: lessons from a rapid autopsy program. Cancer Research 64 9209-9216.

Solit DB, Zheng FF, Drobnjak M, Munster PN, Higgins B, Verbel D, Heller G, Tong W, Cordon-Cardo C, Agus DB, Scher HI \& Rosen N 2002 17-Allylamino-17-demethoxygeldanamycin induces the degradation of androgen receptor and HER-2/neu and inhibits the growth of prostate cancer xenografts. Clinical Cancer Research 8 986-993.

Titus MA, Gregory CW, Ford OH III, Schell MJ, Maygarden SJ \& Mohler JL 2005a Steroid 5-alpha-reductase isozymes I and II in recurrent prostate cancer. Clinical Cancer Research 11 4365-4371.

Titus MA, Schell MJ, Lih FB, Tomer KB \& Mohler JL $2005 b$ Testosterone and dihydrotestosterone tissue levels in recurrent prostate cancer. Clinical Cancer Research 11 4653-4657.
Tran CP, Lin C, Yamashiro J \& Reiter RE 2002 Prostate stem cell antigen is a marker of late intermediate prostate epithelial cells. Molecular Cancer Research 1 113-121.

Unni E, Sun S, Nan B, McPhaul MJ, Cheskis B, Mancini MA \& Marcelli M 2004 Changes in androgen receptor nongenotropic signaling correlate with transition of $\mathrm{LNCaP}$ cells to androgen independence. Cancer Research 64 7156-7168.

Uzgare AR \& Isaacs JT 2004 Enhanced redundancy in Akt and mitogen-activated protein kinase-induced survival of malignant versus normal prostate epithelial cells. Cancer Research 64 6190-6199.

Uzgare AR, Xu Y \& Isaacs JT 2004 In vitro culturing and characteristics of transit amplifying epithelial cells from human prostate tissue. Journal of Cellular Biochemistry 91 196-205.

van Bokhoven A, Varella-Garcia M, Korch C, Johannes WU, Smith EE, Miller HL, Nordeen SK, Miller GJ \& Lucia MS 2003 Molecular characterization of human prostate carcinoma cell lines. Prostate 57 205-225.

Waltregny D, Leav I, Signoretti S, Soung P, Lin D, Merk F, Adams JY, Bhattacharya N, Cirenei N \& Loda M 2001 Androgen-driven prostate epithelial cell proliferation and differentiation in vivo involve the regulation of $\mathrm{p} 27$. Molecular Endocrinology 15 765-782.

Weeraratna AT, Arnold JT, George DJ, De Marzo A \& Isaacs JT 2000 Rational basis for Trk inhibition therapy for prostate cancer. Prostate 45 140-148.

Weeraratna AT, Dalrymple SL, Lamb JC, Denmeade SR, Miknyoczki S, Dionne CA \& Isaacs JT 2001 Pan-trk inhibition decreases metastasis and enhances host survival in experimental models as a result of its selective induction of apoptosis of prostate cancer cells. Clinical Cancer Research 7 2237-2245.

Xu Y, Dalrymple S, Becker R \& Isaacs JT 2006 Pharmacological basis for dutasteride's enhanced efficacy against prostatic cancers. Clinical Cancer Research (In press).

Yan G, Fukabori Y, Nikolaropoulos S, Wang F \& McKeehan WL 1992 Heparin-binding keratinocyte growth factor is a candidate stromal-to-epithelial-cell andromedins. Molecular Endocrinology 6 2123-2128.

Yang Q, Fung K-M, Day WV, Kropp BP \& Lin H-K 2005 Androgen receptor signaling is required for androgensensitive human prostate cancer cell proliferation and survival. Cancer Cell International 58.

Young JC \& Hartl FU 2000 Polypeptide release by Hsp90 involves ATP hydrolysis and is enhanced by the cochaperone p23. EMBO Journal 19 5930-5940.

Zegarra-Moro OL, Schmidt LJ, Huang H \& Tindall DJ 2002 Disruption of androgen receptor function inhibits proliferation of androgen-refractory prostate cancer cells. Cancer Research 62 1008-1013. 